\title{
Comunicação em saúde relacionada à funcionalidade dos idosos.
}

\section{Health communication related to the elderly functionality.}

\section{Comunicación en salud y funcionalidad de los adultos mayores.}

Ryvia Rose Ferraz BEZERRA ${ }^{1}$

Carmem Lúcia Goulart RIBEIRO ${ }^{2}$

Monica REBOUCAS ${ }^{3}$

RESUMO: O estudo apresenta a discussão de pesquisadores que questionaram seu próprio saber ao tempo em que investigaram publicações o que permitiu redefinir categorias e conceitos relativos ao estado funcional dos idosos. Parte-se do marco teórico indicado na CIF-OMS e apreende-se forma de referir-se as pessoas mais velhas. A revisão narrativa da literatura e um grupo focal resultaram em: Síntese de conteúdo - Segundo a CIF, a funcionalidade compõe-se do "Corpo" (subdividido em "Estrutura e Função"); das "Atividades e Participação" as quais o "Corpo" realiza alem dos Fatores Contextuais, ou "Fatores Ambientais", que são circunstâncias em que o "Corpo" realiza "Atividades e Participação" constituídas de ambiente físico, social e atitudinal onde pessoas vivem. Ficha síntese - partindo da síntese de conteúdo foi criada ficha visando à troca de informação sobre funcionalidades dos idosos por meio de uniformização de conceitos propostos por órgão internacional. Então, o estudo apresentou termos que facilitaram a comunicação em saúde ampliando o intercambio de ideias e compreensão sobre a capacidade funcional dos idosos. Palavras chave: Funcionalidade. Idosos. Incapacidade. Linguagem.

\begin{abstract}
The study presents a researchers' argument that questioned about their own knowledge besides investigating publishing which allowed to redefine categories and concepts about the elderly functional state. The theoretical boundary proposed in ICF-WHO suggests a synthetic form of referring to the functionalities of the elderly people. A narrative review of the literature and focus groups resulted in: Synthesis of the content - according to the theoretical boundary proposed in the IFC, the functionality consists of the "Body" (subdivided into "Structure and Function");
\end{abstract}

\footnotetext{
1 dra.ryviarose@gmail.com

2 clgoulart@click21.com.br

3 monicareboucas@oi.com.br
} 
of "Activities and Participation" which the "Body" performs in addition to Contextual Factors or "Environmental Factors" which are circumstances that the "body" performs "Activities and Participation" consisting of the physical, social and attitudinal environment where people live. Summary Sheet - starting from synthesis of content a report was created regarding to the information exchange about functionality of the elderly people by standardizing terms proposed by international agency. Regarding it, the study presented terms that made easier the health communication increasing the interchange of ideas and the understanding about the elderly functional capacity. Keywords: Functionality. Elderly people. Disability. Language.

RESUMEN: Este estudio presenta una discusión entre investigadores que cuestionaron su propio saber al mismo tiempo que investigaron publicaciones, lo que permitió redefinir categorías y conceptos relativos al estados funcional de los adultos mayores. Se parte del marco teórico de la CIF-OMS en cuanto a la forma de referirse a los adultos mayores. La revisión de literatura y un grupo focal resultaron en esta síntesis de contenido: Según la CIF, la funcionalidad se compone de un "Cuerpo" (subdividido en estructura y función), las "Actividades y Participación" que el "Cuerpo" realiza y los factores contextuales o "Factores Ambientales" que son las circunstancias en las cuales el cuerpo realiza las "actividades y participación” constituidas por el ambiente físico, social y actitudinal donde las personas se desenvuelven. Ficha Síntesis - partiendo de la síntesis de contenido fue creada una ficha pensando en el intercambio de información sobre las funcionalidades de los adultos mayores por medio de la uniformización de los conceptos propuestos por el órgano internacional. El estudio presentó términos que facilitaron la comunicación en salud, ampliando el intercambio de ideas y la comprensión sobre la capacidad funcional de los adultos mayores. Palabras clave: Funcionalidad. Adultos mayores. Incapacidad. Lenguaje.

\section{INTRODUÇÃO}

A capacidade funcional vem sendo exaltada diante do reconhecimento de que a saúde pressupõe não somente ausência de doenças, mas manutenção de atividade e de independência em portadores de agravos crônicos ${ }^{1}$.

A capacidade funcional das pessoas expressa-se por meio da realização das atividades básicas da vida diária (ABVDs) e das instrumentais (AIVDs). As primeiras são representadas por atos de autocuidado, como alimentação, controle de esfíncteres, transferência, higiene pessoal ${ }^{2}$. As instrumentais referem-se a atitudes mais complexas, incluindo adaptações ao meio, a exemplo de usar o telefone, fazer compras, usar transporte, tomar remédio, cuidar do dinheiro 3 .

Considerando a importância crescente do tema, publicações à semelhança da Classificação Internacional de Doenças, $\mathrm{CID}^{4}$, tentam fazer o mesmo papel diante das incapacidades em saúde. Algumas dessas publicações, lideradas pela Organização Mundial da Saúde - OMS, são encontradas 
desde $1980^{5,6}$. E confirmando o interesse, a cada publicação são propostos novos modelos, novas nomenclaturas e novas dimensões. A última publicação da OMS sobre o assunto visa estabelecer unificação da linguagem e recebe o título: Classificação Internacional de Funcionalidade, Incapacidade e Saúde, conhecida pelo acrônimo, $\mathrm{CIF}^{7}$.

Este trabalho parte do marco teórico da CIF e apresenta síntese de termos que pode ser usada na clinica de pessoas mais velhas. A sugestão serve aos interessados no envelhecimento populacional que lidam com o aumento do número de indivíduos idosos, portanto, depois da sexta década de vida, em distintos países.

Os objetivos do trabalho foram testar o uso das nomenclaturas proposta pela CIF - $\mathrm{OMS}^{7}$ na abordagem dos idosos e reforçar a comunicação em saúde por meio da linguagem relacionada às funcionalidades.

\section{Desenvolvimento}

A seguir serão descritos os passos executados no estudo.

Foi estabelecida relação metodológica do trabalho com o conteúdo da CIF. A partir desta relação foi realizada revisão de literatura e grupo focal ${ }^{8}$.

Inicialmente a literatura foi revisada de forma narrativa visando identificar publicações sobre a CIF, no idioma português. Os textos foram lidos e discutidos por equipe interdisciplinar formada por médicos geriatra e de família, fisioterapeutas e nutricionista usando a estratégia de grupo focal para desenvolver o tema.

A partir da discussão mencionada em parágrafo anterior foi extraída ficha síntese. A síntese de conteúdo, assim como a ficha síntese, resulta da interpretação da CIF por uma equipe de saúde, com experiência em atendimento a idosos, usando termos encontrados em publicações relacionadas ao tema.

O trabalho norteou os profissionais a formar opiniões e diagnósticos sobre idosos. Portanto, o estudo provocou reflexões na equipe que colheu dados por meio de termos próprios, sugeridos pela OMS, e transformou as informações em conhecimento, reforçando uma efetiva comunicação em saúde.

O teor final serviu a trabalhos de pós-graduação em São Paulo, e foi apresentado em congressos, com reconhecimento dos interessados no tema ${ }^{9-12}$.

\section{(1) Síntese de conteúdo:}

O conteúdo da CIF destaca que as condições de saúde das pessoas se revelam no que elas podem ou não fazer em seu dia-a-dia. Essas condições relacionam-se com estruturas do corpo, funções 
$46 / /$

de órgãos ou sistemas assim como com atividades, participação social e o ambiente em que os indivíduos vivem ${ }^{4}$.

Desta maneira, a CIF conceitua termos como funcionalidade como sendo o que significa a síntese de componentes físicos, mentais, emocionais e, portanto sociais, que permitem a realização de tarefas diárias necessárias à vida independente em comunidade de pessoas humanas ${ }^{13}$. Incapacidades também esta definido na CIF e se relaciona a participação das pessoas na vida cotidiana. As incapacidades são problemas, ou seja, limitações ou restrições das atividades, referidas pelo termo genérico deficiências ${ }^{7}$. As incapacidades, portanto, são relacionadas às deficiências, segundo este enfoque apresentado.

A CIF classifica o tema em seções:

(1.1) A primeira parte da CIF refere-se à Funcionalidade e à Incapacidade, cujos componentes são "Corpo" com subdivisões de "Estrutura e Função" e "Atividades e Participação", que é o que o corpo realiza. Detalhes são citados a seguir:

\section{Estruturas do corpo}

Partes anatômicas do corpo, como órgãos, membros e componentes, a exemplo das estruturas do sistema nervoso; dos olhos, ouvidos e estruturas relacionadas; das relacionadas à voz e à fala; aos sistemas cardiovascular, imunológico, respiratório, digestivo, metabólico, endócrino, geniturinário e reprodutivo; ao movimento assim como a pele e estruturas relacionadas a ela.

\section{Funções do corpo}

Funções fisiológicas dos sistemas corporais, inclusive as psicológicas, a saber, as funções mentais, do cérebro como consciência, energia, impulso, memória, linguagem e cálculo; as sensoriais e dor como dos sentidos visão, audição, paladar, bem como a sensação de dor; as funções da voz, da produção de sons e da fala; dos sistemas cardiovascular, hematológico, imunológico, respiratório, envolvendo o coração, vasos, produção de sangue, imunidade; a respiração, tolerância a exercícios; as dos sistemas digestivo, metabólico, endócrino, como a ingestão, digestão, eliminação, bem como o metabolismo e as glândulas endócrina; as geniturinárias com as funções urinárias, reprodutivas, incluindo as sexuais e de procriação; as funções neuromúsculo esqueléticas relacionadas ao movimento, relacionadas ao movimento e à mobilidade, incluindo as articulações, ossos, reflexos e músculos assim como a pele com suas funções assim como as unhas e pelos.

\section{Atividade}

Reconhece-se que as "Funções e Estruturas do Corpo" dos indivíduos executam tarefas ou ações, as atividades, que podem encontrar limitações. As limitações caracterizam-se em gradações que demandam classificações. 


\section{Participação}

Refere-se a situações vividas por "Funções e Estruturas do Corpo" das pessoas e podem ocorrer restrições no desenvolvimento dessas situações.

\section{(1.2) A outra parte da CIF aborda os Fatores Contextuais, ou "Fatores Ambientais":}

\section{Fatores ambientais}

Constituem circunstâncias em que as "Funções e Estruturas do Corpo" realizam "Atividades e Participação" e compõem o ambiente físico, social e atitudinal onde pessoas vivem.

\section{(1.3) Taxonomia na CIF:}

A CIF identifica em "Funções e Estruturas do Corpo" a ocorrência de problemas ocasionados por limitações em "Atividades" e restrições da "Participação". Esses problemas são classificados por meio de qualificadores. Um dos qualificadores indica a magnitude do problema e caracteriza uma deficiência das "Funções e Estruturas do Corpo" que pode ser graduada em níveis. Além desse qualificador existem outros na CIF, não detalhados nesta ocasião ${ }^{4}$.

\section{Qualificador das deficiências}

Nas gradações NENHUMA deficiência (0 - para nenhuma, ausente, escassa) Deficiência LEVE (1 - leve, baixa); Deficiência MODERADA (2-média, regular); Deficiência GRAVE (3 - alta, extrema) ou Deficiência COMPLETA (4 - total).

Os graus de deficiencia, segundo a CIF, podem acontecer a depender de dispositivos de ajuda ou ajuda de terceiros. Esses aspectos podem se relacionar com o que foi chamado de dificuldades. As deficiências são diferentes se os portadores recebem ou não ajuda, mudando assim suas dificuldades.

Então, na CIF o reconhecimento da necessidade de ajuda ocorre por meio do domínio intitulado dificuldade, que varia de acordo com a escala a seguir.

\section{Qualificador das dificuldades}

Nas gradações NENHUMA dificuldade (0 - para nenhuma, ausente, escassa) Dificuldade LEVE (1 - leve, baixa); Dificuldade MODERADA (2-média, regular); Dificuldade GRAVE (3 - alta, extrema) ou Dificuldade COMPLETA (4 - total).

As "Funções e Estrutura do Corpo", assim como as "Atividades e Participação", podem então ser impedidas por meio de problemas que ocorrem em consequência de alterações advindas de diferentes níveis de fenômenos e por isso são quantificados. 


\section{Quantificador dos problemas}

Nas gradações NÃO há problema (0 - nenhum, ausente, insignificante); Problema LEVE (1 - leve, pequeno); Problema MODERADO (2 - médio, regular); Problema GRAVE (3 - grande, extremo); Problema COMPLETO (4 - total).

\section{(2) Ficha síntese:}

A Síntese do Conteúdo da CIF permitiu que a equipe de saúde construísse uma ficha indutiva.

\section{CONSIDERAÇÕES FINAIS}

A principal contribuição deste texto aponta que o uso do conteúdo proposto na CIF pode servir de instrumento para uma efetiva comunicação em saúde.

O trabalho diz respeito ao que foi desenvolvido em um serviço, em dado momento. A divulgação serve de exemplo e estimula diferentes estratégias de atenção à saúde das pessoas da comunidade. Sua aplicação pressupõe inclusive apontar problemas; consubstanciar crítica e propor solução.

A proposta foi distinguir categorias e, a partir delas, formar conceitos sobre a capacidade funcional, para então defini-las. A ficha síntese insita aos pesquisadores a reconhecer estrutura do corpo; função do corpo; fator ambiental e a necessidade de ajuda para então definir a funcionalidade.

A proposta mais recente, denominada CIF, publicada em português no ano de 2003, avança para além de publicações anteriores ${ }^{4,6}$ já que não define com palavras distintas (desvantagens, deficiência) o fenômeno global da incapacidade ${ }^{14}$.

A publicação mencionada em parágrafo anterior, que na essência define nomenclatura e códigos para identificação relacionada às consequências de doenças humanas, acaba balizando uma forma de comunicação sobre o tema. O volume de 2003 detalha esquematicamente as funções e estruturas do corpo, em suas dimensões físicas, psicológicas e sociais e chama atenção para sua interação com o ambiente, formando um todo, indivisível. Deste conjunto surge a funcionalidade e também o oposto, a incapacidade ${ }^{7}$. Torna-se nítido no texto o esforço em apontar funcionalidade como condição multifatorial ${ }^{7}$.

Entretanto vale ressaltar que são encontradas críticas contundentes ao conteúdo da CIF ${ }^{14}$.

As ressalvas atingem a falta de clareza do texto; os aspectos essencialmente quantitativos assim como sobre seus subsídios ideológicos.

As restrições a CIF podem refletir a contradição implícita no conceito da capacidade funcional: fenômeno subjetivo de caráter biopsicossocial, dependente de bases socialmente construídas, e com inequívocas variações próprias das culturas dos indivíduos. Todavia, as medidas e nomenclaturas 
relativas à capacidade funcional vêm enfocando, em separado, seu aspecto eminentemente físico, de conceito tão complexo, tornando óbvio o insucesso relativo à sua definição e, portanto estabelecimento de nomenclatura. Exalta-se, por exemplo, o forte componente de exclusão dos que possuem incapacidades, ainda não abordado adequadamente em publicações do gênero ${ }^{14}$.

Há de se reconhecer também que publicações, que propõem divulgação e uniformização de termos, podem não esgotar a vasta linguagem aplicada ao tema das incapacidades em saúde, considerando seu amplo impacto social.

E, diante da complexidade do tema, reconhecem-se avanços. Sobretudo porque em desafios como o citado, pressupõe-se intensa discussão prévia ao surgimento da proposta definitiva ${ }^{14}$.

A proposta atual, então, mesmo que controversa, merece aplicação. Principalmente diante dos entraves à comunicação em saúde causados pela variedade de termos usados para relacionar a capacidade funcional das pessoas.

A multiplicidade de termos se torna empecilho considerando que avaliação da funcionalidade recebe atenção de profissionais de saúde em diversificados níveis da prática profissional, assim como na elaboração de pesquisas já que o fenômeno envolve aspectos de mobilidade física, de doenças, das condições sociais e dos componentes psicológicos ${ }^{15}$.

Essa variedade de usuários dos termos relativos à funcionalidade comumente procuram alternativas para integrar suas opiniões, tanto ao realizar avaliação como diagnóstico. Uma alternativa para integração das opiniões dos usuários que atuam na área de saúde tem sido por meio da reunião deles. Neste encontro torna-se mais relevante o uso de palavras ao alcance de todos. Somente assim podem ser conquistados os objetivos de promoção, prevenção e tratamento das pessoas. Na reunião da equipe de saúde ocorre troca de conhecimentos entre várias especialidades e profissões, e construção de consensos, que será alcançado com uso de linguagem comum ${ }^{16}$.

A reunião da equipe de saúde se trata de dinâmica usada inclusive em programa de amplo espectro como o Saúde da Família (PSF) formulada pelo Ministério da Saúde do Brasil (MS) em 1993. Os dados relacionados ao PSF revelam que já foram atendidas mais de 100 milhões de pessoas e beneficiadas suas famílias por meio de ações preventivas ${ }^{17}$. Neste programa o método de realizar visita domiciliar segue-se de reunião da equipe como forma de uniformizar procedimentos a serem usados na população, pressupondo discurso comum entre os componentes. $\mathrm{O}$ dialogo constante implícito na reunião da equipe do PSF serve de exemplo do quanto à comunicação relacionada à saúde se torna estratégica do ponto de vista de implantação da gestão e das políticas públicas ${ }^{18}$.

A equipe envolve agentes comunitários de saúde assim como outros profissionais engajados na busca do entendimento para a prestação da melhor assistência, por meio de compartilhar saberes e integrar interesses ${ }^{16}$ também quando se trata de incapacidades, justificando trabalhos, como este, 
que exalta a unificação da terminologia facilitando a comunicação em saúde.

Lembre-se que a estratégia de atuar em equipe, já citada, ultrapassa esses limites e pode ser útil inclusive no preenchimento de instrumentos e na avaliação da saúde de idosos, como o que foi apresentado neste trabalho. A prática de reunir grupo de profissionais de saúde para discutir temas tem se tornado comum e nesses encontros os termos interpretados em separado recebem a visão do conjunto e permitem a construção "do todo" que compõe a funcionalidade dos idosos. Se interpretados em equipe os distintos elementos da funcionalidade funcionam como indicadores uns dos outros e seu significado pode ser decifrado a depender do que se deseja obter.

Considerando o aumento da ocorrência das incapacidades nos com seis ou mais décadas de vida, a equipe de saúde interessada nos que envelhecem acaba envolvida tanto na avaliação como no diagnóstico das funcionalidades dessas pessoas, necessitando assim referir-se ao tema da capacidade funcional dos mais velhos ${ }^{13}$. Termos propostos por órgão internacional ${ }^{7}$ se revelam uteis, ao facilitar a comunicação de ideias contidas em programas de atenção aos idosos, facilitando trocas de informações sobre suas funcionalidades.

A atenção à saúde dos idosos vem sendo mais desenvolvida recentemente o que estimula a divulgação de experiências, como esta aqui apresentada, para que sirva de consulta dos interessados, principalmente quando publicadas em periódico.

O estudo aqui apresentado revela um marco teórico resultante da discussão de pesquisadores, usuários, que ao contrário do que se esperava, questionaram seu próprio saber, investigaram publicações, redefiniram categorias e conceitos.

Em síntese, o estudo apresentou esforço de aprimoramento da comunicação em saúde ampliando o intercambio de ideias e compreensão sobre tema relacionado à funcionalidade. $\mathrm{O}$ movimento caminha na direção de diminuir a proliferação de nomenclaturas relativas às incapacidades e facilita o reconhecimento dos fatores críticos e de estratégias para manutenção da vida saudável dos indivíduos, inclusive os mais velhos, moradores da comunidade.

\section{REFERÊNCIAS BIBIOGRÁFICAS}

1. Ramos LR. Fatores determinantes do envelhecimento saudável em idosos residentes em centros urbanos: Projeto Epidoso. Cad. Saúde Pública. São Paulo. 2003; 19(3): 793-98.

2. Lino, VTS, Pereira SEM, Camacho LAB, Ribeiro Filho ST, Buksman S. Adaptação transcultural da escala de independência em atividades da vida diária (Escala de Katz). Cad Saúde Pública. 2008; 24 (1): 103-12.

3. Paschoal SMP. Autonomia e Independência. In: Papaleo NM. Gerontologia: A Velhice e o envelhecimento em visão globalizada. São Paulo: Atheneu; 2002; Cap. 28:313-23. 
4. Faria N, Buchalla CM. A classificação internacional de funcionalidade, incapacidade e saúde da organização mundial de saúde: conceitos, usos e perspectivas. Rev Bras Epidemiol. 2005; 8(2): 187-93.

5. Araujo ESD. A classificação internacional de funcionalidade, incapacidade e saúde (CIF) em fisioterapia: uma revisão bibliográfica [dissertação]. São Paulo: Universidade de São Paulo, Faculdade de Saúde Pública; 2008.

6. Buuren SV, Hopman-Rock M, Revision of the ICIDH severity of disabilities scale by data linking and item response theory. Statist Med. 2011; 20: 1061-76.

7. Organização Mundial da Saúde. Classificação Internacional de Funcionalidade, Incapacidade e Saúde. São Paulo: Editora da Universidade de São Paulo - EDUSP; 2003:325.

8. Minayo, MCS. O desafio do Conhecimento - Pesquisa Qualitativa em Saúde. São Paulo: Editora Hucitec; 2007.

9. Bezerra RRF. Alterações do corpo e necessidade de ajuda em atividades diárias de idosos da Vila Clementino na cidade de São Paulo no ano de 2009 [Monografia]. São Paulo: Centro Universitário São Camilo; 2011.

10. Bezerra RRF, Rebouças M. Pôster- Linguagem proposta na CIF aplicada à gerontologia. In: GERP, $7^{\circ}$. Congresso Paulista de Geriatria e Gerontologia; 2011 Abril 13-16; Santos, Brasil.

11. Silva CLGR. Ergonomia domiciliar e dependência em atividades de vida diária de idosos em São Paulo, ano 2009 [Monografia]. São Paulo: Centro Universitário São Camilo; 2011.

12. Rebouças M. Estudo de confiabilidade e validade das questões sobre atividades da vida diária em idosos brasileiros [tese]. São Paulo: Universidade Federal de São Paulo, Escola Paulista de Medicina; 2012.

13. Alves LC, Leite IDC, Machado CJ. Conceituando e mensurando a incapacidade funcional da população idosa: uma revisão de literatura. Ciência \& Saúde Coletiva. 2008; 13(4): 1199-207.

14. Mângia EF, Muramoto MT, Lancman S. Classificação Internacional de Funcionalidade e Incapacidade e Saúde (CIF): Processo de Elaboração e Debate sobre a questão da incapacidade. Rev. Ter. Ocup. Univ. São Paulo. 2008 maio/ago; 19(2): 121-30.

15. Brito FC, Nunes MI, Yuaso DR. Multidimensionalidade em Gerontologia II: Instrumentos de Avaliação. In: Papaleo NM, organizador. Tratado de Gerontologia. São Paulo: Atheneu; 2007; Cap. 10: 133-47. 
16. Ribeiro EM, Pires D, Blank VLG. A teorização sobre processo de trabalho em saúde como instrumental para a análise do trabalho no Programa Saúde da Família. Cad. Saúde Pública. 2004 mar/abr; 20(2): 438-46.

17. Ministério da Saúde. Brasil. Portal da Saúde. [citado 10 mai. 2010]. Disponível em: http:// dab.saude.gov.br/atencaobasica.php\#saudedafamilia.

18. Giacomozzi CM, Lacerda MR. A Prática da Assistência Domiciliar dos Profissionais da Estratégia de Saúde da Família. Texto Contexto Enferm, Florianópolis, 2006 Out-Dez; 15(4): 64553.

Artigo apresentado em 10/02/14

Artigo aprovado em 14/07/14

Artigo publicado no sistema em 22/10/14 